\title{
Assessing cost-effectiveness of HPV vaccines with decision analytic models: what are the distinct challenges of low- and middle-income countries? A protocol for a systematic review
}

\author{
Obinna I. Ekwunife ${ }^{1,2^{*}}$, Andreas Gerber Grote ${ }^{3}$, Christoph Mosch ${ }^{4}$, James F. O'Mahony ${ }^{5}$ and Stefan K. Lhachimi ${ }^{1,6}$
}

\begin{abstract}
Background: Cervical cancer poses a huge health burden, both to developed and developing nations, making prevention and control strategies necessary. However, the challenges of designing and implementing prevention strategies differ for low- and middle-income countries (LMICS) as compared to countries with fully developed health care systems. Moreover, for many LMICs, much of the data needed for decision analytic modelling, such as prevalence, will most likely only be partly available or measured with much larger uncertainty. Lastly, imperfect implementation of human papillomavirus (HPV) vaccination may influence the effectiveness of cervical cancer prevention in unpredictable ways. This systematic review aims to assess how decision analytic modelling studies of HPV cost-effectiveness in LMICs accounted for the particular challenges faced in such countries. Specifically, the study will assess the following: (1) whether the existing literature on cost-effectiveness modelling of HPV vaccines acknowledges the distinct challenges of LMICS, (2) how these challenges were accommodated in the models, (3) whether certain parameters systemically exhibited large degrees of uncertainty due to lack of data and how influential were these parameters on model-based recommendations, and (4) whether the choice of modelling herd immunity influences model-based recommendations, especially when coverage of a HPV vaccination program is not optimal.
\end{abstract}

Methods: We will conduct a systematic review to identify suitable studies from MEDLINE (via PubMed), EMBASE, NHS Economic Evaluation Database (NHS EED), EconLit, Web of Science, and CEA Registry. Searches will be conducted for studies of interest published since 2006. The searches will be supplemented by hand searching of the most relevant papers found in the search. Studies will be critically appraised using Consolidated Health Economic Evaluation Reporting Standards (CHEERS) statement checklist. We will undertake a descriptive, narrative, and interpretative synthesis of data to address the study objectives.

Discussion: The proposed systematic review will assess how the cost-effectiveness studies of HPV vaccines accounted for the distinct challenges of LMICs. The gaps identified will expose areas for additional research as well as challenges that need to be accounted for in future modelling studies.

Systematic review registration: PROSPERO CRD42015017870.

Keywords: Uterine cervical neoplasm, Papillomavirus vaccines, Mass vaccination, Cost-effectiveness analysis, Low- and middle-income countries

\footnotetext{
* Correspondence: ekwunife@uni-bremen.de

${ }^{1}$ Cooperative Research Group for Evidence-Based Public Health, Department

of Prevention and Evaluation, Leibniz Institute for Prevention Research and

Epidemiology—BIPS, Bremen, Germany

${ }^{2}$ Department of Clinical Pharmacy and Pharmacy Management, Nnamdi

Azikiwe University, Awka, Nigeria

Full list of author information is available at the end of the article
}

C Biomed Central (c) 2015 Ekwunife et al.; licensee BioMed Central. This is an Open Access article distributed under the terms of the Creative Commons Attribution License (http://creativecommons.org/licenses/by/4.0), which permits unrestricted use, distribution, and reproduction in any medium, provided the original work is properly credited. The Creative Commons Public Domain Dedication waiver (http://creativecommons.org/publicdomain/zero/1.0/) applies to the data made available in this article, unless otherwise stated. 


\section{Background}

Cervical cancer poses one of the greatest challenges to women's health. It is estimated that over a million women worldwide currently have cervical cancer, most of whom have not been diagnosed nor have access to treatment that could cure them or extend their survival [1]. The burden of cervical cancer is disproportionately borne by poorer countries. In 2012, 528,000 new cases of cervical cancer were diagnosed, and 266,000 women died of the disease, nearly $90 \%$ of them in low- and middle-income countries (LMICs) [1]. It is expected that these numbers will double in the next 20 years due to aging and population growth [2].

The human papillomavirus (HPV) is the primary cause of cervical cancer and cervical intraepithelial neoplasia (CIN) [3]. HPV is typically transmitted in the cervix through microabrasions that may occur as a result of sexual intercourse [4]. Persistent infection with oncogenic strains of HPV causes cervical cancer [5]. Of the $40 \mathrm{HPV}$ strains that affect the genital area, 15 of them are known to be oncogenic (types 16, 18, 31, 33, 35, 39, $45,51,52,56,58,59,68,73$, and 82) [6]. HPV infection has also been linked with other genital cancers (e.g., vaginal, vulvar, anal, and penile) as well as non-lifethreatening diseases, such as genital warts [7]. HPV types 16 and 18 have been found to be responsible for about $70 \%$ of all cervical cancer cases worldwide $[8,9]$. In the remaining $30 \%$ of cervical cancers, HPV types 31 , $33,35,45$, and 58 have all been implicated [10].

Cervical cancer can be prevented both by primary prevention through HPV vaccination and by secondary prevention through cervical cancer screening. Conventional cytology-based screening, combined with colposcopy and treatment of precursor lesions, has been the gold standard for the secondary prevention of cervical cancer. It has greatly reduced cervical cancer incidence and mortality from cervical cancer in many developed countries. Cytology-based screening is not as widespread in developing countries [10]. For instance, it is estimated that less than $5 \%$ of women at risk of cervical cancer in sub-Saharan Africa have ever been screened [11].

Alternatives to cytology-based screening include visual inspection with acetic acid (VIA) and newer molecular testing for infection of the cervix with high-risk types of HPV DNA. VIA and HPV DNA testing have proven to be effective screening methods $[12,13]$. HPV DNA testing has shown to be significantly more effective than VIA or cytology in reducing both cervical cancer precursors and cervical cancer $[12,13]$.

There currently are two HPV vaccines in widespread use. Both Cervarix ${ }^{\circ}$ and Gardasil $^{\circ}$ offer protection against HPV 16 and 18, respectively, the two most oncogenic types [7]. Gardasil $^{{ }^{-}}$also offers protection against HPV 6 and 11, which cause $90 \%$ of genital warts [14]. The quadrivalent vaccine has also shown to protect against cancers of the anus, vagina, and vulva [14]. There is evidence indicating that the immune response against type 16 and 18 provides some cross-protection against types 45 and 31, both important in the aetiology of cervical cancer, thus potentially increasing the projected protection from vaccination to $75-80 \%$ [10]. However, since prophylactic vaccination is not effective against infection from all 15 oncogenic HPV types, regular screening is still recommended among women that have received the vaccination [14].

\section{Challenges of prevention and control strategies in low- and middle-income countries}

The World Health Organization's (WHO) recommendation for comprehensive cervical cancer prevention and control strategy includes primary, secondary, and tertiary prevention activities [1]. Primary prevention involves HPV vaccination of girls (and boys if affordable) between 9 to 13 years. In secondary prevention, women 30 years of age or older are to be "screened-and-treated" with low-cost technology, e.g., VIA followed by cryotherapy or HPV testing for highrisk HPV type. For tertiary prevention, all women with invasive cancer at any age are to be treated with ablative surgery, radiotherapy, or chemotherapy with palliative care as necessary. The recommendation suggests that the three prevention components be planned and implemented in combination with a structured national approach to community education and mobilization strategies and a national monitoring and evaluation system [1].

LMICs potentially face a number of challenges in implementing such a cervical cancer prevention and control program. Existing health services may not have the capacity to accommodate additional interventions, and thus, extra cost will be incurred to set up such a program. Resource constraints may necessitate implementation of a program in phases rather than immediate implementation. Resource constraints could also require the introduction of preventive and treatment services only for certain regions rather than country-wide. Sections of the population may systematically avoid participation in such a program. Given these potential challenges, it is therefore important to examine if and how they are accounted for by decision analytic models of HPV vaccines; for example, do analyses adopt a different modelling structure or just change parameters for the constraints mentioned above?

\section{Data challenges in low- and middle-income countries}

The data needed for modelling might be less readily available in LMICs or measured with much larger uncertainty. HPV-related outcome data in many LMICs may have poor resolution and are also often highly aggregated [15]. For example, incidence data of cervical cancer available from the WHO/Institut Català d'Oncologia Information Center on HPV and Cervical Cancer is stratified into the age groups 
$0-14,15-44,45-54,55-64$, and $\geq 65$ years, and thus, simple natural history models may erroneously predict that 15-year-old women have the same cancer incidence as 44year-old women, and this overestimates the proportion of cancers occurring in younger women and thus potentially biases estimates of vaccination cost-effectiveness [15]. These data challenges prompt the questions of how well they are recognized and overcome by the existing literature and which of these uncertain parameters have the greatest impact on the model outcomes. These are pertinent questions, and it is worthwhile examining how they have been handled in modelling studies of HPV vaccination.

\section{Model type and herd immunity}

There are three types of models that have been employed in cost-effectiveness analyses of HPV vaccination: static models, transmission dynamic models, and hybrid models combining features of both static and dynamic models [7]. A static model typically tracks progression of HPV disease for a single cohort over an expected lifetime [16]. Transmission dynamic models have the advantage of accounting for both the direct and indirect (i.e., herd immunity) benefits of vaccination in the population [16]. Thus, dynamic models account for the immunity that occurs when HPV vaccination of a significant portion of the population (or herd) provides a measure of protection for individuals who have not been vaccinated. A hybrid model is a combination of a cohort model and a dynamic model. It corrects the invariant incidence probability in a cohort model to a dynamic probability and thus does not ignore the indirect benefits of herd immunity for the cohort being simulated [16]. However, herd immunity depends on the rate of vaccine coverage. As stated previously, this coverage may vary dramatically in LMICs, both across time or across populations. It is therefore relevant to ask how this is accounted for in HPV vaccine cost-effectiveness studies.

\section{Study rationale and objectives}

Appraising the introduction of HPV vaccines requires estimation of the avertable disease burden, costeffectiveness of the vaccine compared with alternative uses of the resources, affordability of the vaccine, feasibility of achieving high coverage, likelihood of public acceptability, and political support for vaccination [17]. The lack of data on the long-term effectiveness of HPV vaccination has prompted the development of various decision analytic models to guide policy makers by projecting the long-term epidemiologic and economic consequences of alternative vaccination policies [16]. For such analyses to provide a reliable guide to policy development and implementation, they should reflect the LMIC-specific challenges described above.

Two systematic reviews of cost-effectiveness analyses of HPV vaccination in LMICs have been published previously
$[18,19]$. These reviews discussed the cost-effectiveness estimates and investigated how they are affected by model characteristics and underlying assumptions in general. The focus of our proposed systematic review is to examine how the modelling studies accounted for the challenges specific to low- and middle-income countries. The review seeks to answer the following questions:

- Does the existing literature on cost-effectiveness modelling of HPV vaccine acknowledge the particular challenges facing LMICs?

- How were particular challenges accommodated in the models, e.g., through a different model structure or just by varying parameters?

- Is the uncertainty among the less readily available essential data/parameters regarding LMICs so large that the model-based recommendation is affected?

- Does the choice of modelling herd immunity influence model-based recommendations, and in particular, does imperfect HPV vaccination coverage influence model-based recommendations?

\section{Methods/design}

\section{Protocol}

This protocol adheres to the Preferred Reporting Items in Systematic Reviews and Meta-analyses (PRISMA) statement [20]. The protocol is registered in the International Prospective Register of Systematic Reviews (PROSPERO) CRD42015017870.

\section{Eligibility criteria}

Inclusion criteria are as follows:

- Studies based on decision analytical models of HPV vaccination;

- Studies that considered the cost-effectiveness of HPV vaccination and reported the additional cost and additional health effects in terms of life years gained (LYGs), quality-adjusted life years (QALYs), or disability-adjusted life year (DALYs);

- LMICs as identified by the World Bank classification of income groups [21];

- Both single- and multi-country studies;

- The review will include both original research papers and reviews (inclusion of the latter is to ensure that no original study was missed);

- Studies to be included in the review could be published in any language;

- Studies published since 2006.

\section{Information sources}

We will search MEDLINE (via PubMed), EMBASE, NHS Economic Evaluation Database (NHS EED), EconLit, Web of Science, and Tufts CEA Registry. For existing systematic 
reviews, we will search Cochrane Reviews, Cochrane Database of Abstracts of Reviews of Effects (DARE), and Cochrane Health Technology Assessment Databases. Reviews will be included to reduce the possibility of missing an individual study.

\section{Search strategy}

A search strategy will be developed for each of the databases. The "Appendix" section provides details of our planned bibliographic database search strategies for MEDLINE (via PubMed), EMBASE, CINAHL, Cochrane Reviews/Cochrane DARE/NHS EED, EconLit, Web of Science, and CEA Registry. The reference lists of all included and relevant articles identified during the search will be reviewed to identify further studies that were missed. Furthermore, we will use the PubMed "related articles" feature. Hand searching of a selection of relevant journals will be conducted as advised by experts in economic evaluation.

\section{Study selection}

Titles and abstracts will be screened for inclusion independently by two of the reviewers using the eligibility criteria. Opinion of a third reviewer will be sought to arrive at a consensus in case of disagreement on a study for inclusion.

\section{Data extraction}

Data will be extracted independently by two of the reviewers from included studies using a predefined data extraction spreadsheet (Table 1). Data to be extracted will be arranged into the following classes: model characteristics, base-case assumptions, results, sensitivity/uncertainty analyses, data sources, and miscellaneous (conflict of interests and factors not considered). Cost presented in different currencies will be adjusted to 2013 value using consumer price index. Afterwards, cost data will be converted to international dollar units using purchasing power parities (PPPs). Authors of various studies may be contacted to clarify methods and results if the need arises.

\section{Risk of bias and data synthesis}

One of the investigators will assess the validity of included studies using the Consolidated Health Economic Evaluation Reporting Standards (CHEERS) statement [22]. Details of the CHEERS statement are summarized in Table 2. Descriptive, narrative, and interpretative synthesis of data will be undertaken to address the study objectives [23]. The WHO Commission on Macroeconomics and Health will be used to determine the thresholds of cost-effectiveness such that an intervention will be considered "very cost effective" and "cost effective" when its incremental cost-effectiveness ratio is less than the gross domestic product (GDP) per capita and less than three times GDP per capita, respectively [23].

\section{Discussion}

This protocol describes a systematic review for studies on cost-effectiveness of prophylactic HPV vaccination in LMICs. The aim is to assess how cost-effectiveness studies of HPV vaccine accounted for the individual challenges of low- and middle-income countries. The gaps identified in this systematic review will expose areas for additional research as well as challenges that need to be accounted for in future modelling studies. The review will also present current data on cost-effectiveness of HPV vaccines in LMICs as newer studies have been carried out since the publication of the last reviews.

\section{Appendix: Details of electronic bibliographic database search strategies MEDLINE (via PubMed)}

("Human papillomavirus 6" [mesh] OR "Human papillomavirus 16" [mesh] OR "Human papillomavirus 18" [mesh] OR "Human papillomavirus 31" [mesh] OR Alphapapillomavirus [mesh] OR Papillomavirus" [tiab] OR human papilloma* [tiab] OR HPV [tiab] OR "Papillomavirus Infections" [mesh] OR Papillomaviridae [mesh] OR Papillomavirid" [tiab] OR Uterine Cervical Neoplasms [mesh] OR cervix cancer" [tiab] OR cervix carcinom* [tiab] OR cervix malignan* [tiab] OR cervix neoplas" [tiab] OR cervix tumor* [tiab] OR cervical cancer* [tiab] OR cervical carcinom* [tiab] OR cervical malignan* [tiab] OR cervical neoplas* [tiab] OR cervical tumor* [tiab] OR Cervical Intraepithelial Neoplasia [mesh] OR Cervical Intraepithelial Neoplasia [tiab] OR CIN [tiab])

AND

(Vaccination [mesh] OR mass vaccination [mesh] OR Papillomavirus Vaccines [mesh] OR vaccin* [tiab] OR immunization [mesh] OR Immunization Programs [mesh] OR immuni" [tiab] OR "human papillomavirus vaccine L1, type 6,11,16,18" [Supplementary Concept] OR "human papillomavirus vaccine, L1 type 16, 18" [Supplementary Concept] OR Gardasil [tiab] OR Cervarix [tiab])

AND

(“2006/01/01”[dp] : “3000”[dp])

AND

(Economics [mesh] OR Quality of Life [mesh:NoExp] OR Value of Life [mesh:NoExp] OR Quality-Adjusted Life Years [mesh:NoExp] OR Models, Economic [mesh:NoExp] OR Markov Chains [mesh:NoExp] OR Monte Carlo Method [mesh:NoExp] OR Decision trees [mesh:NoExp] OR economic* [tiab] OR cost" [tiab] OR costing* [tiab] OR costly [tiab] OR costed [tiab] OR price* [tiab] OR pricing* [tiab] OR pharmacoeconomic* [tiab] OR pharmaco-economic* [tiab] OR budget* [tiab] OR expenditure* [tiab] OR (value [tiab] AND (money [tiab] 
Table 1 Relevant data extraction information

\begin{tabular}{|c|c|}
\hline Data extraction category & Specific information to extract \\
\hline \multicolumn{2}{|l|}{ A. Model characteristics } \\
\hline Model & $\begin{array}{l}\text { Author(s), year of publication, model design (static model, dynamic model, } \\
\text { or hybrid model) }\end{array}$ \\
\hline Perspective & Providers, patients, or societal perspective \\
\hline Benefits (QALY, DALY, YLS) & $\begin{array}{l}\text { Measured in quality-adjusted life years (QALYs), disability-adjusted life years } \\
\text { (DALY), years of life saved (YLS) }\end{array}$ \\
\hline \multicolumn{2}{|l|}{ B. Base-case assumptions } \\
\hline Current routine practice efficacy & $\begin{array}{l}\text { Efficacy of the current screening practice (cytology-based screening, DNA } \\
\text { screening, or visual inspection with acetic acid) used as the comparator }\end{array}$ \\
\hline Screening age/screening interval & Age at which women commence screening and screening interval \\
\hline Vaccine coverage in target groups & Current HPV vaccination coverage \\
\hline Age for vaccination & Age group eligible to receive HPV vaccination \\
\hline Estimated effective coverage & HPV vaccination coverage and the rationale for the assumption \\
\hline Screening compliance & Estimate used for comparison with current practice \\
\hline Sensitivity/specificity of the screening & Sensitivity/specificity estimate for the screening methods \\
\hline Duration of vaccine protection & $\begin{array}{l}\text { Total length of time HPV vaccination is assumed to protect the recipient } \\
\text { from acquiring infection }\end{array}$ \\
\hline Cost of vaccine per three doses (and booster if included) & $\begin{array}{l}\text { Market cost or subsidized cost. Other cost associated with vaccination, e.g., } \\
\text { freight, storage, and program cost. School-based-delivery cost or } \\
\text { health-facility-based delivery cost }\end{array}$ \\
\hline Discounting rate & Discounting rate used to adjust for time preference for money \\
\hline \multicolumn{2}{|l|}{ C. Results } \\
\hline Incremental cost-effectiveness ratio (ICER) & The most cost-effective protocol compared with the second best protocol \\
\hline Year based for currency value & The year which the analysis was conducted \\
\hline Adjusted ICER (to 2014) & Adjusted ICER to reflect 2014 value \\
\hline \multicolumn{2}{|l|}{ Data sources } \\
\hline Source(s) & $\begin{array}{l}\text { Data source used to derive estimates of HPV-related epidemiologic outcomes, } \\
\text { e.g., HPV (type-specific) prevalence, cervical cancer incidence, probability of } \\
\text { HPV transmission given a sexual partnership, and crude mortality from } \\
\text { cervical cancer. }\end{array}$ \\
\hline \multicolumn{2}{|l|}{ D. Sensitivity and uncertainty } \\
\hline Sensitivity analysis & Parameters that had the highest effect on model-based recommendation. \\
\hline Uncertainty analysis & The contribution of individual parameters on overall uncertainty (when reported). \\
\hline \multicolumn{2}{|l|}{ E. Miscellaneous } \\
\hline Funding and conflict of interest & $\begin{array}{l}\text { Funding for the study and the role of the funder in the study. Possible conflict } \\
\text { of interest declared by the author }\end{array}$ \\
\hline Factors not taken into account & $\begin{array}{l}\text { How did model acknowledge and account for special challenges of the LMIC? } \\
\text { Choice of modelling herd immunity }\end{array}$ \\
\hline
\end{tabular}

OR monetary [tiab])) OR fee [tiab] OR fees [tiab] OR quality of life [tiab] OR qol* [tiab] OR hrqol* [tiab] OR quality adjusted life year* [tiab] OR qaly* [tiab] OR cba [tiab] OR cea [tiab] OR cua [tiab] OR utilit* [tiab] OR markov* [tiab] OR monte carlo [tiab] OR (decision [tiab] AND (tree* [tiab] OR analys* [tiab] OR model* [tiab])) OR ((clinical [tiab] OR critical [tiab] OR patient [tiab]) AND (path* [tiab] OR pathway* [tiab])) OR (managed [tiab] AND (care [tiab] OR network* [tiab])))

\section{(just) EMBASE}

('Human papillomavirus type 6'/exp OR 'Human papillomavirus type 16 ' exp OR 'Human papillomavirus type 18'/exp OR 'Human papillomavirus type 31'/exp OR 'Alphapapillomavirus'/exp OR 'Papillomavirus Infection'/ exp OR 'Papilloma virus'/exp OR 'uterine cervix cancer'/ exp OR (Papillomavirus* OR human papilloma* OR HPV OR Papillomavirid*):ab,ti OR ((cervix OR cervical) NEAR/3 (cancer* OR carcinoma* OR malignanc* OR 
Table 2 CHEERS statement for checking the validity of included studies [22]

\begin{tabular}{|c|c|c|}
\hline Selection/item & Item no. & Recommendation \\
\hline \multicolumn{3}{|l|}{ Title and abstract } \\
\hline Title & 1 & $\begin{array}{l}\text { Identify the study as an economic evaluation, or use more specific } \\
\text { terms such as "cost-effectiveness analysis" and describe the } \\
\text { interventions compared. }\end{array}$ \\
\hline Abstract & 2 & $\begin{array}{l}\text { Provide a structured summary of objectives, perspective, setting, } \\
\text { methods (including study design and inputs), results (including } \\
\text { base-case and uncertainty analyses), and conclusions. }\end{array}$ \\
\hline \multicolumn{3}{|l|}{ Introduction } \\
\hline Background and objectives & 3 & $\begin{array}{l}\text { Provide an explicit statement of the broader context for the study. } \\
\text { Present the study question and its relevance for health policy or } \\
\text { practice decisions. }\end{array}$ \\
\hline \multicolumn{3}{|l|}{ Methods } \\
\hline Target population and subgroups & 4 & $\begin{array}{l}\text { Describe characteristics of the base-case population and subgroups } \\
\text { analyzed including why they were chosen. }\end{array}$ \\
\hline Setting and location & 5 & $\begin{array}{l}\text { State relevant aspects of the system(s) in which the decision(s) } \\
\text { need(s) to be made. }\end{array}$ \\
\hline Study perspective & 6 & $\begin{array}{l}\text { Describe the perspective of the study and relate this to the costs } \\
\text { being evaluated. }\end{array}$ \\
\hline Comparators & 7 & $\begin{array}{l}\text { Describe the interventions or strategies being compared and state } \\
\text { why they were chosen. }\end{array}$ \\
\hline Time horizon & 8 & $\begin{array}{l}\text { State the time horizon(s) over which costs and consequences are } \\
\text { being evaluated and say why appropriate. }\end{array}$ \\
\hline Discount rate & 9 & $\begin{array}{l}\text { Report the choice of discount rate(s) used for costs and outcomes } \\
\text { and say why appropriate. }\end{array}$ \\
\hline Choice of health outcomes & 10 & $\begin{array}{l}\text { Describe what outcomes were used as the measure(s) of benefit in } \\
\text { the evaluation and their relevance for the type of analysis performed. }\end{array}$ \\
\hline \multirow[t]{2}{*}{ Measurement of effectiveness } & $11 a$ & $\begin{array}{l}\text { Single study-based estimates: describe fully the design features of the } \\
\text { single effectiveness study and why the single study was a sufficient } \\
\text { source of clinical effectiveness data. }\end{array}$ \\
\hline & $11 b$ & $\begin{array}{l}\text { Synthesis-based estimates: describe fully the methods used for the } \\
\text { identification of included studies and synthesis of clinical } \\
\text { effectiveness data. }\end{array}$ \\
\hline Measurement and valuation of preference-based outcomes & 12 & $\begin{array}{l}\text { If applicable, describe the population and methods used to elicit } \\
\text { preference outcomes. }\end{array}$ \\
\hline \multirow[t]{2}{*}{ Estimating resources and costs } & $13 a$ & $\begin{array}{l}\text { Single study-based economic evaluation: describe approaches used to } \\
\text { estimate resource use associated with the alternative interventions. } \\
\text { Describe primary or secondary research methods for valuing each } \\
\text { resource item in terms of its unit cost. Describe any adjustments } \\
\text { made to approximate to opportunity costs. }\end{array}$ \\
\hline & $13 b$ & $\begin{array}{l}\text { Model-based economic evaluation: describe approaches and data } \\
\text { sources used to estimate resource use associated with model health } \\
\text { states. Describe primary or secondary research methods for valuing } \\
\text { each resource item in terms of its unit cost. Describe any adjustments } \\
\text { made to approximate to opportunity costs. }\end{array}$ \\
\hline Currency, price date, and conversion & 14 & $\begin{array}{l}\text { Report the dates of the estimated resource quantities and unit costs. } \\
\text { Describe methods for adjusting estimated unit costs to the year of } \\
\text { reported costs if necessary. Describe methods for converting costs } \\
\text { into a common currency base and the exchange rate. }\end{array}$ \\
\hline Choice of mode & 15 & $\begin{array}{l}\text { Describe and give reasons for the specific type of decision analytic } \\
\text { model used. Providing a figure to show model structure is } \\
\text { strongly recommended. }\end{array}$ \\
\hline Assumptions & 16 & $\begin{array}{l}\text { Describe all structural or other assumptions underpinning the decision } \\
\text { analytic model. }\end{array}$ \\
\hline
\end{tabular}


Table 2 CHEERS statement for checking the validity of included studies [22] (Continued)

\begin{tabular}{|c|c|c|}
\hline Analytic methods & 17 & $\begin{array}{l}\text { Describe all analytic methods supporting the evaluation. This could } \\
\text { include methods for dealing with skewed, missing, or censored data; } \\
\text { extrapolation methods; methods for pooling data; approaches to } \\
\text { validate or make adjustments (e.g., half-cycle corrections) to a model; } \\
\text { and methods for handling population heterogeneity and uncertainty. }\end{array}$ \\
\hline \multicolumn{3}{|l|}{ Results } \\
\hline Study parameters & 18 & $\begin{array}{l}\text { Report the values, ranges, references, and, if used, probability distributions } \\
\text { for all parameters. Report reasons or sources for distributions used to } \\
\text { represent uncertainty where appropriate. Providing a table to show the } \\
\text { input values is strongly recommended. }\end{array}$ \\
\hline Incremental costs and outcomes & 19 & $\begin{array}{l}\text { For each intervention, report mean values for the main categories of } \\
\text { estimated costs and outcomes of interest, as well as mean differences } \\
\text { between the comparator groups. If applicable, report incremental } \\
\text { cost-effectiveness ratios. }\end{array}$ \\
\hline \multirow[t]{2}{*}{ Characterizing uncertainty } & $20 a$ & $\begin{array}{l}\text { Single study-based economic evaluation: describe the effects of sampling } \\
\text { uncertainty for estimated incremental cost, incremental effectiveness, } \\
\text { and incremental cost-effectiveness, together with the impact of } \\
\text { methodological assumptions (such as discount rate, study perspective). }\end{array}$ \\
\hline & $20 b$ & $\begin{array}{l}\text { Model-based economic evaluation: describe the effects on the results of } \\
\text { uncertainty for all input parameters and uncertainty related to the } \\
\text { structure of the model and assumptions }\end{array}$ \\
\hline \multicolumn{3}{|l|}{ Discussion } \\
\hline $\begin{array}{l}\text { Study findings, limitations, generalizability, } \\
\text { and current knowledge }\end{array}$ & 22 & $\begin{array}{l}\text { Summarize key study findings and describe how they support the } \\
\text { conclusion reached. Discuss limitation and the generalizability of the } \\
\text { findings and how the findings fit with current knowledge. }\end{array}$ \\
\hline \multicolumn{3}{|l|}{ Others } \\
\hline Source of funding & 23 & $\begin{array}{l}\text { Describe how the study was funded and the role of the funder in the } \\
\text { identification, design, conduct, and reporting of the analysis. Describe } \\
\text { other non-monetary sources of support. }\end{array}$ \\
\hline Conflicts of interest & 24 & $\begin{array}{l}\text { Describe any potential for conflict of interest among study contributors } \\
\text { in accordance with journal policy. In the absence of a journal policy, we } \\
\text { recommend authors comply with International Committee of Medical } \\
\text { Journal Editors' recommendations. }\end{array}$ \\
\hline
\end{tabular}

neoplasm* OR tumor*)):ab,ti OR 'uterine cervix carcinoma in situ'/exp OR ("Cervical Intraepithelial Neoplasia” OR CIN):ab,ti)

\section{AND}

('Immunization'/exp OR 'Cancer immunization'/exp OR 'mass immunization'/exp OR 'Vaccination'/exp OR 'Wart virus vaccine'/exp OR (vaccin* OR immuni" OR Gardasil OR Cervarix):ab,ti OR 'preventive health service'/exp)

AND

\section{[1-1-2006]/sd NOT [1-1-3000]/sd}

\section{AND}

('health economics'/exp OR 'health care cost'/exp OR 'quality of life'/exp OR 'quality adjusted life year'/exp OR 'Monte Carlo method'/exp OR 'decision tree'/exp OR (economic* OR cost* OR costing* OR costly OR costed OR price* OR pricing* OR pharmacoeconomic* OR budget* OR expenditure* OR fee OR fees OR 'quality of life' OR qol* OR hrqol* OR qaly* OR CBA OR CEA OR CUA OR utilit* OR markov*):ab,ti OR (pharmaco NEXT/1 economic*):ab,ti OR (value NEAR/1 (money OR monetary)):ab,ti OR ('quality adjusted life'
NEXT/1 year*):ab,ti OR (monte NEXT/1 carlo):ab,ti OR (decision NEXT/3 (tree* OR analys* OR model*)):ab,ti OR ((clinical OR critical OR patient) NEXT/1 (path* OR pathway*)):ab,ti OR (managed NEXT/3 (care OR network $\left.\left.\left.{ }^{*}\right)\right): a b, t i\right)$

\section{EconLit and CINAHL (via EBSCO)}

MH Papilloma OR MH Papillomaviruses OR MH "papillomavirus infections" OR AB (papilloma OR papillomavirus* OR HPV) OR MH "cervix neoplasms" OR MH "uterine neoplasms" OR AB ((cancer* OR carcinoma* OR malignanc* OR neoplasm* OR tumor*) N3 (uterine OR cervix OR cervical)) OR MH "Cervical Intraepithelial Neoplasia" OR AB CIN AND MH "papillomavirus vaccine" OR MH immunization OR MH "Immunization Programs" OR AB (immuni?ation OR vaccin* OR gardasil OR cervarix) AND PD (2006 OR 2007 OR 2008 OR 2009 OR 201\%) AND MH Health Resource Allocation OR MH Health Care Delivery OR MH Health Care Costs OR MH Economic Value of Life OR MH Quality of Life OR MH Quality-Adjusted Life Years OR MH Decision Trees OR AB (economic* OR cost* OR costing* 
OR costly OR costed OR pri?e* OR pri?ing* OR pharmacoeconomic* OR budget" OR expenditure* OR fee OR fees OR "quality of life" OR qol* OR hrqol* OR qaly* OR CBA OR CEA OR CUA OR utilit* OR markov*) OR AB ((pharmaco N1 economic*) OR (value N1 (money OR monetary)) OR ("quality adjusted life" N1 year") OR (monte N1 carlo) OR (decision N3 (tree* OR analys* OR model*)) OR ((clinical OR critical OR patient) N1 (path* OR pathway*)) OR (managed N3 (care OR network*)))

\section{Cochrane Reviews/Cochrane DARE/NHS EED/HTA database}

MeSH descriptor: [Human papillomavirus 6] explode all trees OR MeSH descriptor: [Human papillomavirus 16] explode all trees OR MeSH descriptor: [Human papillomavirus 18] explode all trees OR MeSH descriptor: [Human papillomavirus 31] explode all trees OR $\mathrm{MeSH}$ descriptor: [Alphapapillomavirus] explode all trees OR $\mathrm{MeSH}$ descriptor: [Papillomaviridae] explode all trees OR MeSH descriptor: [Papillomavirus Infections] explode all trees OR MeSH descriptor: [Uterine Cervical Neoplasms] explode all trees OR MeSH descriptor: [Uterine Neoplasms] explode all trees OR MeSH descriptor: [Cervical Intraepithelial Neoplasia] explode all trees OR (Papillomavirus* OR human papilloma* OR HPV OR Papillomavirid* OR ((cervix OR cervical) NEAR/3 (cancer* OR carcinoma* OR malignanc* OR neoplasm* OR tumor*)) OR "Cervical Intraepithelial Neoplasia" OR CIN):ti,ab,kw

AND

MeSH descriptor: [Papillomavirus Vaccines] explode all trees OR MeSH descriptor: [Immunization] explode all trees OR MeSH descriptor: [Vaccination] explode all trees OR MeSH descriptor: [Mass Vaccination] explode all trees OR MeSH descriptor: [Immunization Programs] explode all trees OR (vaccin* OR immuni* OR Gardasil OR Cervarix):ti,ab,kw

AND

MeSH descriptor: [Economics] explode all trees OR $\mathrm{MeSH}$ descriptor: [Economics, Medical] explode all trees OR MeSH descriptor: [Economics, Pharmaceutical] explode all trees OR MeSH descriptor: [Health Care Economics and Organizations] explode all trees OR MeSH descriptor: [Health Care Costs] explode all trees OR $\mathrm{MeSH}$ descriptor: [quality of life] explode all trees OR MeSH descriptor: [value of life] explode all trees OR MeSH descriptor: [Quality-Adjusted Life Years] explode all trees OR MeSH descriptor: [Markov Chains] explode all trees OR MeSH descriptor: [Monte Carlo method] explode all trees OR MeSH descriptor: [decision trees] explode all trees OR (economic* OR cost* OR costing* OR costly OR costed OR price* OR pricing* OR pharmacoeconomic $^{*}$ OR budget* OR expenditure* OR fee OR fees OR 'quality of life' OR qol" OR hrqol" OR qaly" OR CBA OR CEA OR CUA OR utilit* OR markov** OR (pharmaco NEXT/1 economic*) OR (value NEAR/1 (money OR monetary)) OR ('quality adjusted life' NEXT/1 year*) OR (monte NEXT/1 carlo) OR (decision NEXT/3 (tree* OR analys* OR model*)) OR ((clinical OR critical OR patient) NEXT/1 (path* OR pathway*)) OR (managed NEXT/3 (care OR network*))):ti,ab,kw

AND

Limit Publication Date: 01-1-2006 till now

\section{Web of Science (Core Collection)}

TS = ((cervix OR cervical) NEAR/3 (cancer* OR carcinoma* OR malignanc" OR neoplasm* OR tumor*)) OR Papillomavirus* OR human papilloma* OR HPV OR Papillomavirid" OR "Cervical Intraepithelial Neoplasia" OR CIN)

\section{AND}

TS $=\left(\right.$ vaccin$^{*}$ OR immuni* OR Gardasil OR Cervarix $)$ AND

PY $=(2006-2015) \quad$ AND TS $=$ (health econom $*$ OR health care cost* OR Monte Carlo OR economic* OR cost* OR costing* OR costly OR costed OR pri? e* OR pri?ing* OR pharmacoeconomic* OR pharmaco economic* OR budget* OR expenditure* OR fee OR fees OR quality of life OR quality adjusted life year* OR qol* OR hrqol* OR qaly* OR CBA OR CEA OR CUA OR utilit* OR markov* OR (value NEAR/1 (money OR monetary OR life)) OR (decision NEXT/3 (tree* OR analys* OR model*)) OR ((clinical OR critical OR patient) NEAR/1 (path* OR pathway*)) OR (managed NEAR/3 $($ care OR network*)))

\section{CEA Registry}

Basic/manual search by the following keywords (published 2006-2015): Papilloma, Papillomavirus, Papillomaviridae, HPV 74, cervix, cervical, uterine, Gardasil and Cervari

\section{Abbreviations}

CHEERS: Consolidated Health Economic Evaluation Reporting Standards; CIN: Cervical intraepithelial neoplasia; DALYs: Disability-adjusted life years; GDP: Gross domestic product; HPV: Human papillomavirus; LMICs: Low- and middle-income countries; LYGs: Life years gained; NHS EED: NHS Economic Evaluation Database; PRISMA: Preferred Reporting Items in Systematic Reviews and Meta-analyses; VIA: Visual inspection with acetic acid; WHO: World Health Organization.

\section{Competing interests}

The authors declare that they have no competing interests.

\section{Authors' contributions}

OE and SKL were responsible for the development of the study design, formulation of study questions, study selection criteria, synthesis of data, and risk of bias assessments. AGG and JFOM contributed in the development of the study design and study selection criteria. CM was responsible for formulating the search. OE was responsible for compiling of the table and data extraction spreadsheet. OE drafted the first manuscript, and all the authors contributed to manuscript revisions before the final version. All authors read and approved the final manuscript. 


\section{Authors' information}

OE is currently a postdoctoral fellow with the Research Group for Evidence-based Public Health, (BIPS GmbH/Universität Bremen). He is a lecturer in the Department of Clinical Pharmacy and Pharmacy Management, Nnamdi Azikiwe University in Nigeria. JFOM is a research fellow in the Department of Health Policy and Management, School of Medicine, Trinity College Dublin, Dublin, Ireland. SKL is the group head of the Research Group for Evidence-based Public Health (BIPS GmbH/University of Bremen). CM is a scientific assistant in the Institute for Research in Operative Medicine (IFOM, Universität Witten/Herdecke). AGG is an adjunct professor of health economics at the University of Cologne.

\section{Acknowledgements}

This study is supported by Georg Forster Research Fellowship received by OE. Georg Forster Research Fellowship is funded by the Alexander von Humboldt Foundation (AVH). AVH had no role in the development of this protocol.

\section{Author details}

${ }^{1}$ Cooperative Research Group for Evidence-Based Public Health, Department of Prevention and Evaluation, Leibniz Institute for Prevention Research and Epidemiology_BIPS, Bremen, Germany. ${ }^{2}$ Department of Clinical Pharmacy and Pharmacy Management, Nnamdi Azikiwe University, Awka, Nigeria. ${ }^{3}$ Institute for Quality and Efficiency in Health Care (IQWiG), Cologne, Germany. ${ }^{4}$ Institute for Research in Operative Medicine (IFOM), Universität Witten/Herdecke, Witten, Germany. ${ }^{5}$ Department of Health Policy and Management, School of Medicine, Trinity College Dublin, Dublin, Ireland. ${ }^{6}$ Institute for Public Health and Nursing Research_IPP, Health Sciences Bremen, University of Bremen, Bremen, Germany.

Received: 31 March 2015 Accepted: 5 May 2015

Published online: 12 May 2015

\section{References}

1. WHO. Comprehensive cervical cancer: a guide to essential practice. Geneva, Switzerland: WHO Press; 2014

2. De Vuyst H, Alemany L, Lacey C, Chibwesha CJ, Sahasrabuddhe V, Banura C, et al. The burden of human papillomavirus infections and related diseases in sub-Saharan Africa. Vaccine. 2013;31 Suppl 5:F32-46. doi:10.1016/j.vaccine.2012.07.092.

3. Bosch FX, Burchell AN, Schiffman M, Giuliano AR, de Sanjose S, Bruni L, et al Epidemiology and natural history of human papillomavirus infections and type-specific implications in cervical neoplasia. Vaccine. 2008;26 Suppl 10:K1-16. doi:10.1016/j.vaccine.2008.05.064.

4. Stanley M, Lowy DR, Frazer I. Chapter 12: prophylactic HPV vaccines: underlying mechanisms. Vaccine. 2006;24 Suppl 3:S3/106-13. doi:10.1016/j.vaccine.2006.05.110.

5. Bosch FX, Lorincz A, Munoz N, Meijer CJ, Shah KV. The causal relation between human papillomavirus and cervical cancer. J Clin Pathol. 2002:55(4):244-65.

6. Munoz N, Bosch FX, de Sanjose S, Herrero R, Castellsague X, Shah KV, et al. Epidemiologic classification of human papillomavirus types associated with cervical cancer. N Engl J Med. 2003;348(6):518-27. doi:10.1056/ NEJMoa021641.

7. Armstrong EP. Prophylaxis of cervical cancer and related cervical disease: a review of the cost-effectiveness of vaccination against oncogenic HPV types. J Manag Care Pharm. 2010;16(3):217-30.

8. de Sanjose S, Quint WG, Alemany L, Geraets DT, Klaustermeier JE, Lloveras B, et al. Human papillomavirus genotype attribution in invasive cervical cancer: a retrospective cross-sectional worldwide study. Lancet Oncol. 2010;11(11):1048-56. doi:10.1016/s1470-2045(10)70230-8.

9. Li N, Franceschi S, Howell-Jones R, Snijders PJ, Clifford GM. Human papillomavirus type distribution in 30,848 invasive cervical cancers worldwide: variation by geographical region, histological type and year of publication. Int J Cancer. 2011;128(4):927-35. doi:10.1002/ijc.25396.

10. Adefuye PO, Broutet NJ, de Sanjose S, Denny LA. Trials and projects on cervical cancer and human papillomavirus prevention in sub-Saharan Africa. Vaccine. 2013;31 Suppl 5:F53-9. doi:10.1016/j.vaccine.2012.06.070.

11. Sankaranarayanan R, Budukh AM, Rajkumar R. Effective screening programmes for cervical cancer in low- and middle-income developing countries. Bull World Health Organ. 2001;79(10):954-62.
12. Denny L, Kuhn L, Hu CC, Tsai WY, Wright Jr TC. Human papillomavirus-based cervical cancer prevention: long-term results of a randomized screening trial. J Natl Cancer Inst. 2010;102(20):1557-67. doi:10.1093/jnci/djq342.

13. Sankaranarayanan R, Nene BM, Shastri SS, Jayant K, Muwonge R, Budukh AM, et al. HPV screening for cervical cancer in rural India. N Engl J Med. 2009;360(14):1385-94. doi:10.1056/NEJMoa0808516.

14. CDC. HPV vaccine information for clinicians - fact sheet. Division of STD Prevention, National Center for HIV/AIDS, Viral Hepatitis, STD, and TB Prevention, Centers for Disease Control and Prevention. 2012. http://www.cdc.gov/std/hpv/ stdfact-hpv-vaccine-hcp.htm. Accessed 11 December 2014.

15. Jit M, Demarteau N, Elbasha E, Ginsberg G, Kim J, Praditsitthikorn N, et al. Human papillomavirus vaccine introduction in low-income and middle-income countries: guidance on the use of cost-effectiveness models. BMC medicine. 2011;9:54. doi:10.1186/1741-7015-9-54.

16. Dasbach EJ, Elbasha EH, Insinga RP. Mathematical models for predicting the epidemiologic and economic impact of vaccination against human papillomavirus infection and disease. Epidemiol Rev. 2006;28:88-100. doi:10.1093/epirev/mxj006.

17. Awolude OA, Morhason-Bello IO, Denny LA, Adewole IF. Human papillomavirus infection and related cancers in sub-Saharan Africa: burden and tools for prevention. Vaccine. 2013;31 Suppl 5:vii-x. doi:10.1016/j.vaccine.2012.06.065.

18. Fesenfeld M, Hutubessy R, Jit M. Cost-effectiveness of human papillomavirus vaccination in low and middle income countries: a systematic review. Vaccine. 2013;31(37):3786-804. doi:10.1016/j.vaccine.2013.06.060.

19. Lehtinen Ta NK. Cost-effectiveness of HPV-vaccination in medium or low income countries with high cervical cancer incidence - a systematic review. Journal of Vaccines \& Vaccination. 2013;04(01). doi:10.4172/2157-7560.1000172.

20. Liberati A, Altman DG, Tetzlaff J, Mulrow C, Gotzsche PC, loannidis JP, et al. The PRISMA statement for reporting systematic reviews and meta-analyses of studies that evaluate health care interventions: explanation and elaboration. PLoS Med. 2009;6(7):e1000100. doi:10.1371/journal.pmed.1000100.

21. World-Bank. Country and Lending Groups. World Bank Group. 2015. http://data.worldbank.org/about/country-and-lending-groups. Accessed 6 January 2015.

22. Husereau D, Drummond M, Petrou S, Carswell C, Moher D, Greenberg D, et al. Consolidated Health Economic Evaluation Reporting Standards (CHEERS)-explanation and elaboration: a report of the ISPOR Health Economic Evaluation Publication Guidelines Good Reporting Practices Task Force. Value Health. 2013;16(2):231-50. doi:10.1016/j.jval.2013.02.002

23. WHO. The World Health Report 20022002.

\section{Submit your next manuscript to BioMed Central and take full advantage of:}

- Convenient online submission

- Thorough peer review

- No space constraints or color figure charges

- Immediate publication on acceptance

- Inclusion in PubMed, CAS, Scopus and Google Scholar

- Research which is freely available for redistribution 